\title{
Luxação intra-articular horizontal da patela: Relato de caso*
}

\section{Horizontal Intraarticular Dislocation of the Patella: Case Report*}

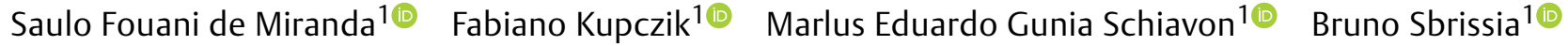 \\ Gustavo Yuiti Kaneko Suzuki ${ }^{1}$ \\ ${ }^{1}$ Serviço de Ortopedia e Traumatologia, Hospital Universitário Cajuru, \\ Curitiba, Brasil \\ Endereço para correspondência Saulo Fouani de Miranda, MD, Rua \\ dos Gerânios, 718, Maringá, PR, 87060158, Brasil \\ (e-mail: s_fm@hotmail.com).
}

Rev Bras Ortop 2020;55(6):800-803.

\section{Resumo \\ Palavras-chave \\ - patela \\ - articulação do joelho \\ - luxação patelar}

Luxação intra-articular da patela é uma afecção incomum, podendo ocorrer deslocamento no plano horizontal ou vertical; pode se associar com lesão/ruptura do tendão do quadríceps, total ou parcial, com lesão osteocondral ou dos ligamentos colaterais. Fatores de risco são: frouxidão ligamentar, hipermobilidade patelar, patela alta e displasia troclear, e é mais comum em jovens, podendo necessitar de redução cruenta. Casos de luxação intra-articular da patela são eventos raros, sendo necessários uma suspeição diagnóstica e um manejo diferenciado. O objetivo deste trabalho é relatar um caso raro de luxação intra-articular da patela, assim como seu quadro clínico, diagnóstico, conduta e revisão da literatura.

Intraarticular dislocation of the patella is an uncommon condition, with displacement in the horizontal or vertical plane, which may be associated with total or partial lesion/rupture of the quadriceps tendon, with osteochondral or collateral ligament injury. Risk factors are ligament laxity, patellar hypermobility, high patella, and trochlear dysplasia. This condition is more common in young people and may require blood reduction. Cases of intraarticular dislocation of the patella are rare events, requiring diagnostic suspicion and differentiated management. The aim of the present study is to report a rare case of intraarticular dislocation of the patella, as well as its clinical condition, diagnosis, management and literature review.

\section{Introdução}

Luxação da patela é uma emergência com incidência anual de 5.8 por cada 100.000 indivíduos. Acomete mais comumente os adolescentes entre 10 e 17 anos que praticam esportes. ${ }^{1} \mathrm{O}$ tipo mais comum de luxação é a lateral, sendo raros os casos de luxação da patela intra-articular. Na luxação horizontal, que é a

\footnotetext{
* Trabalho desenvolvido no Grupo de Cirurgia do Joelho, Serviço de Ortopedia e Traumatologia do Hospital Universitário Cajuru, Curitiba, PR, Brasil.
}

recebido

01 de Setembro de 2019 aceito

12 de Dezembro de 2019
DOI https://doi.org/

$10.1055 / \mathrm{s}-0040-1708516$ ISSN 0102-3616. mais comum, a patela gira sobre seu eixo horizontal, deixando sua superfície articular voltada para superior ou inferior, e na luxação vertical, a patela gira e a superfície articular fica voltada para medial ou lateral. ${ }^{2}$ Descrevemos um caso pouco comum de luxação intra-articular horizontal da patela em um paciente idoso.

\section{Relato de caso}

Uma mulher de 68 anos de idade procurou atendimento devido a um trauma no joelho direito após queda do mesmo

Copyright $\odot 2020$ by Sociedade Brasileira License terms de Ortopedia e Traumatologia. Published by Thieme Revinter Publicações Ltda, Rio de Janeiro, Brazil 


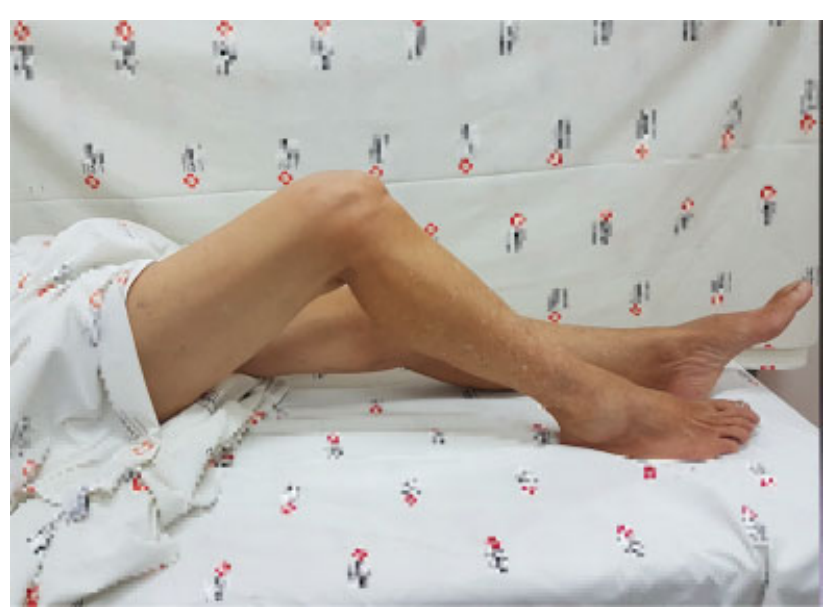

Fig. 1 Exame clínico de admissão.

nível. No momento do acidente, a paciente referiu ter realizado uma hiperflexão do membro inferior direito e uma hiperextensão de membro inferior esquerdo. Ao tentar se levantar, notou uma deformidade no joelho direito com limitação da sua flexo-extensão, além de dor e limitação funcional para deambular.

No exame da admissão, a paciente apresentava dor e aumento de volume no joelho direito, além de um gap na região supra-patelar, mantendo a articulação fletida em $40^{\circ}$ (-Fig. 1). As extensões ativa e passiva apresentavam-se limitadas e era possível realizar a palpação da região intercondilar do fêmur direito, com dificuldade da palpação o polo superior da patela.

As radiografias ântero-posterior e perfil do joelho direito evidenciaram uma patela baixa, com a superfície articular em contato com o espaço intercondilar do fêmur, sugerindo uma luxação intra-articular com face articular para distal/inferior (-Fig. 2). Como investigação complementar foi realizada uma tomografia computadorizada com reconstrução 3D óssea e de partes moles, que não evidenciou fraturas e descartou inicialmente lesões do mecanismo extensor, com apenas uma imagem mostrando uma suspeita

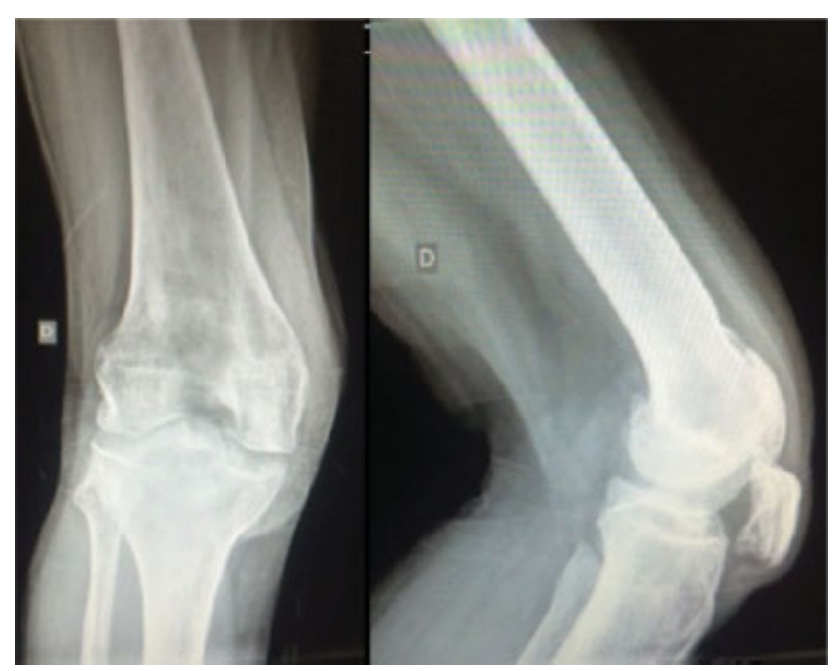

Fig. 2 Radiografias iniciais joelho direito. de lesão parcial das fibras do retináculo lateral ( - Fig. 3), uma ressonância magnética do joelho direito também confirmou a integridade dos tendões patelar e quadricipital (-Fig. 4).

Houve falha na tentativa de manipulação para redução da luxação na sala de emergência, mesmo após a realização de analgesia com $5 \mathrm{mg}$ de morfina intravenosa. Foi optado por levar a paciente ao centro cirúrgico para realizar redução sob sedação, que foi facilmente realizada com um mecanismo de extensão progressiva com manipulação do polo inferior da patela. Na sequência, foi testada a estabilidade da articulação através da flexo-extensão dinâmica, a qual demonstrou-se estável porém com ressaltos e crepitação durante a extensão. A paciente foi mantida com uma imobilização gessada inguinomaleolar. A radiografia pós-redução mostou uma vista tópica, com sinais sugestivos de osteoartrose avançada do compartimento patelo-femoral e lesão osteocondral ( - Fig. 5).

O exame físico pós-redução demonstrou mecanismo extensor de joelho direito preservado. A escolha da realização de ressonância nuclear magnética do joelho direito foi feita como meio de investigação complementar e registro do caso, e não evidenciou ruptura do tendão do quadríceps.

O tratamento foi mantido com imobilização com uma órtese inguinomaleolar, com restrição de carga no membro, analgesia, crioterapia e acompanhamento ambulatorial para reabilitação.

\section{Discussão}

Luxação intra-articular da patela é uma afecção incomum, podendo ocorrer deslocamento no plano horizontal ou verti$\mathrm{cal}^{3}{ }^{3}$ A primeira ocorre após trauma direito com rotação da patela em torno de seu eixo horizontal, podendo a face articular estar voltada para superior ou inferior, há uma rotação interna do fêmur em relação a tíbia, com um rápido movimento de flexão e rotação externa do fêmur e uma força constante no polo superior da patela o que faz com que ela se desloque para a fossa intercondilar. ${ }^{4}$ Pode se associar com lesão/ruptura do tendão do quadríceps, podendo ser total ou parcial, com lesão osteocondral ou dos ligamentos colaterais, necessitando de um exame complementar de imagem, como uma ressonância magnética, para confirmação diagnóstica. ${ }^{4,5}$

O segundo tipo é mais raro, com poucos casos descritos na literatura. O mecanismo de trauma mais comum é um estresse em valgo importante. com o joelho em extensão associado a rotação interna da tíbia. ${ }^{2} \mathrm{~A}$ face articular da patela pode estar voltada para medial ou lateral. ${ }^{5}$ A lesão com desvio do polo superior da patela para a região intra-articular é seis vezes mais frequente que o desvio da região inferior. ${ }^{6}$

A literatura nos traz que este tipo de luxação ocorre mais frequentemente em adolescentes de até 16 anos, sendo incomun em idosos. ${ }^{7-9}$ Outros fatores de risco associados são frouxidão ligamentar, hipermobilidade patelar, patela alta e displasia troclear. Idosos que apresentam alterações degenerativas devido a comorbidades, tais como artrite reumatoide, diabetes mellitus, doença renal crônica e uso crônico de corticoides, que se associam ao enfraquecimento ou ruptura do quadríceps, também são mais susceptíveis a esta patologia. ${ }^{1}$ 


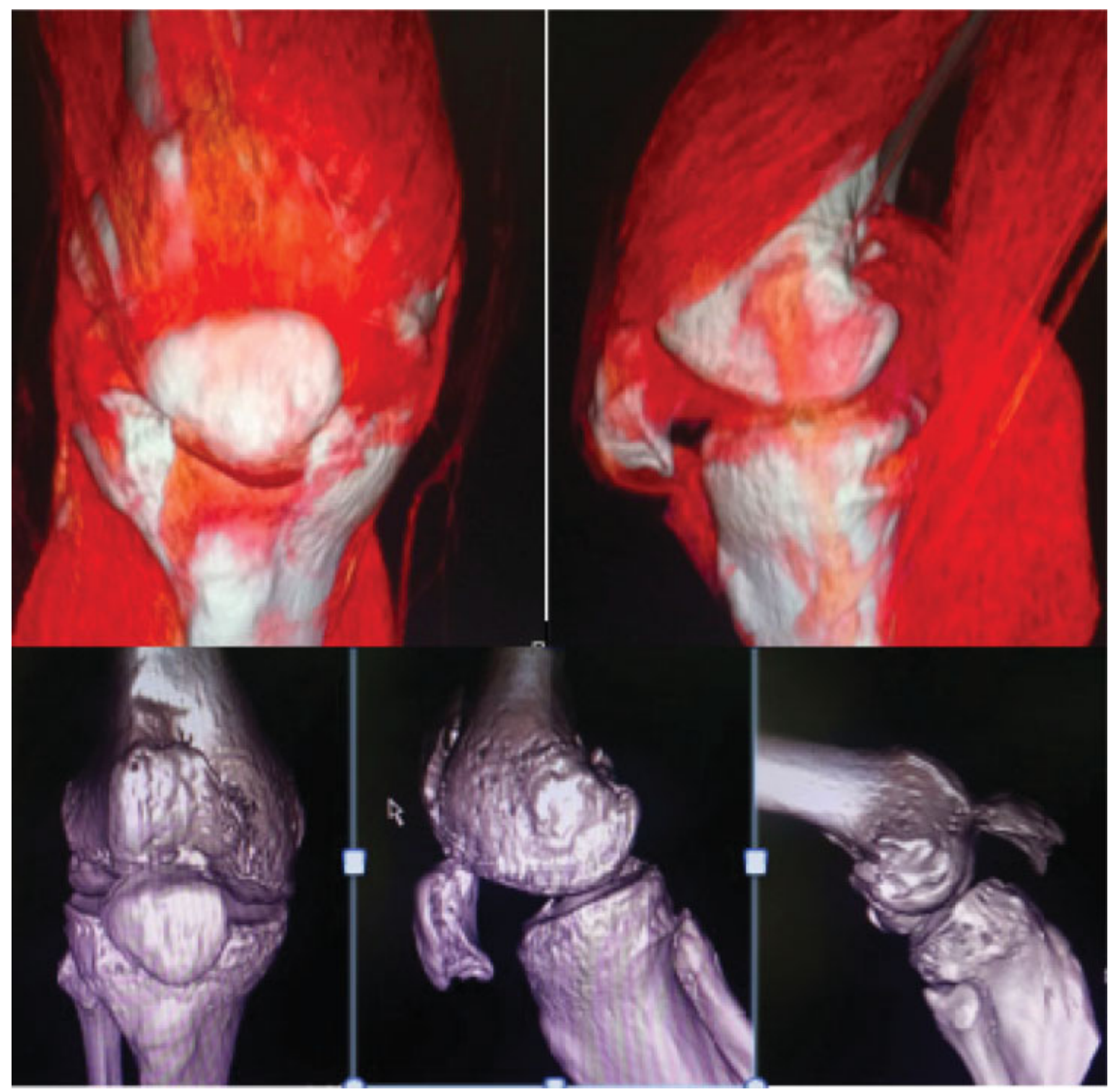

Fig. 3 Reconstrução óssea e de partes moles joelho direito.

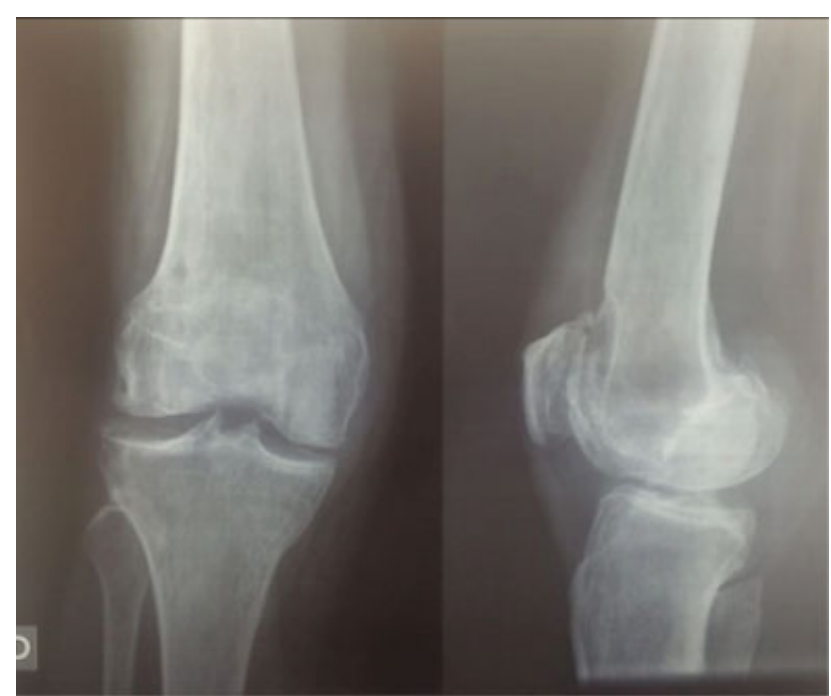

Fig. 4 Radiografias de controle pós-redução.
A maioria desses casos pode requerer redução aberta, sendo raros os casos descritos com redução fechada, ou incruenta, sob anestesia. ${ }^{5}$ Choudhary e Tice $^{6}$ sugeriram que se a rotação da patela for menor que 90 graus, uma redução fechada é passível de ser realizada. Alioto e Kates ${ }^{1}$ descreveram necessidade de tratamento cirúrgico com pino de Schanz após falha de redução fechada e McHugh et al. ${ }^{2}$ realizaram redução aberta por meio da incisão longitudinal do tendão do quadríceps seguida de tração em um paciente idoso com luxação crônica da patela.

Foi realizada imobilização por cerca de 2 semanas, seguida de reabilitação precoce com a recuperação da força e mobilidade pré-lesão. Devido a possibilidade de lesões associadas como, ligamentar, osteocondral ou tendinosa, o desfecho a longo prazo pode não ser tão favorável, necessitando de acompanhamento ambulatorial por tempo mais prolongado, em casos de tratamento conservador. $^{7}$ 


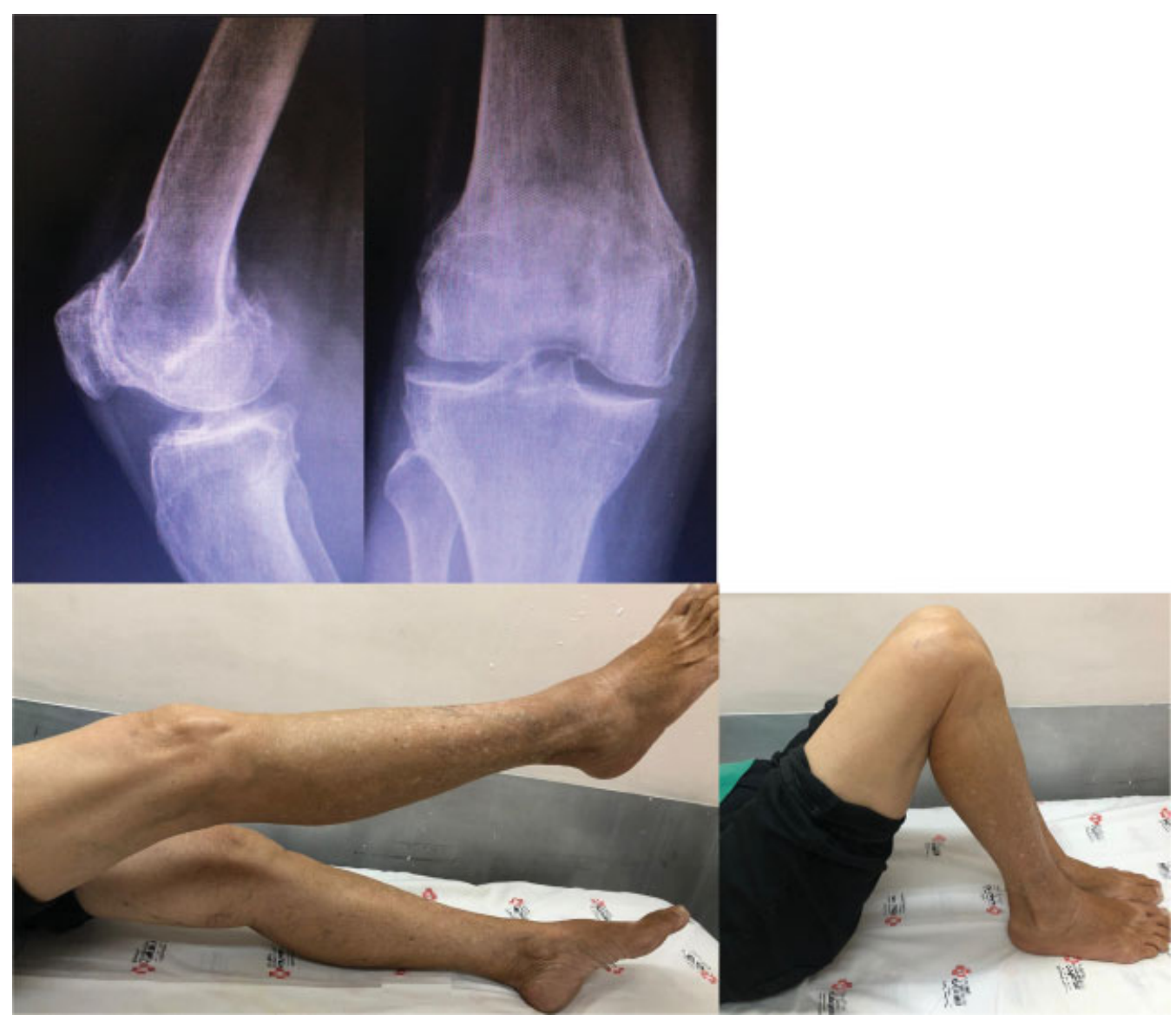

Fig. 5 Radiografias de controle e amplitude de movimento após 2 anos de evolução.

É importante conhecer, estar atento e levantar a suspeita diagnóstica de lesões incomuns como essa, otimizando assim o tratamento e a reabilitação do paciente. O prognóstico costuma ser bom, com recuperação do nível de mobilidade satisfatória.

\section{Conflito de Interesses}

Os autores declaram não haver conflito de interesses.

\section{Referências}

1 Alioto RJ, Kates S. Intra-articular vertical dislocation of the patella: a case report of an irreducible patellar dislocation and unique surgical technique. J Trauma 1994;36(02):282-284

2 McHugh G, Ryan E, Cleary M, Kenny P, O’Flanagan S, Keogh P. Intra-articular dislocation of the patella. Case Rep Orthop 2013; 2013:535803
3 Frangakis EK. Intra-articular dislocation of the patella. A case report. J Bone Joint Surg Am 1974;56(02):423-424

4 Yuguero M, Gonzalez JA, Carma A, Huguet J. Intra-articular patellar dislocation. Orthopedics 2003;26(05):517-518

5 Feneley RC. Intra-articular dislocation of the patella. Report of a case. J Bone Joint Surg Br 1968;50(03):653-655

6 Choudhary RK, Tice JW. Intra-articular dislocation of the patella with incomplete rotation-two case reports and a review of the literature. Knee 2004;11(02):125-127

7 Garner JP, Pike JM, George CD. Intra-articular dislocation of the patella: two cases and literature review. J Trauma 1999;47(04): 780-783

8 Theodorides A, Guo S, Case R. Intraarticular dislocation of patella: a case report and review of literature. Inj Extra 2010; 41:103-105

9 Colville J. An unusual case of intra-articular dislocation of the patella. Injury 1978;9(04):321-322 